\title{
Systematic uncertainty of first-principle calculations of the radi- ation energy emitted by extensive air showers
}

\author{
Marvin Gottowik ${ }^{1, \star}$, Christian Glaser ${ }^{2,3}$, Tim Huege ${ }^{4,5}$, and Julian Rautenberg ${ }^{1}$ \\ ${ }^{1}$ Bergische Universität Wuppertal, Gaußstraße 20, 42119 Wuppertal, Germany \\ ${ }^{2}$ RWTH Aachen University, III. Physikalisches Institut A, Aachen, Germany \\ ${ }^{3}$ Department of Physics and Astronomy, University of California, Irvine, USA \\ ${ }^{4}$ Karlsruher Institut für Technologie, Institut für Kernphysik, Postfach 3640, 76021 Karlsruhe, Germany \\ ${ }^{5}$ Vrije Universiteit Brussel, Astrophysical Institute, Pleinlaan 2, 1050 Brussels, Belgium
}

\begin{abstract}
The energy of extensive air showers can be determined from the energy radiated in the form of radio signals. The so-called radiation energy can be predicted with modern simulation codes using first-principle calculations without the need of free parameters. Here, we verify the consistency of radiation energy calculations by comparing a large set of Monte Carlo simulations made with the two codes CoREAS and ZHAireS. For the frequency band of $30-80 \mathrm{MHz}$, typically used by many current radio detectors, we observe a difference in the radiation energy prediction of $5.2 \%$. This corresponds to a radio emission modelling uncertainty of $2.6 \%$ for the determination of the absolute cosmic-ray energy scale. Hence, radio detection offers the opportunity for a precise, accurate and independent measurement of the absolute energy of cosmic rays.
\end{abstract}

\section{Introduction}

The broadband radio emission from extensive air showers allows a derivation of the properties of the primary cosmic ray particle. The energy emitted in the form of radio signals (radiation energy, $E_{\text {rad }}$ ) can be used for an accurate determination of the absolute cosmic-ray energy scale [1,2]. In simulations, the radio emission can be calculated by applaying classical electrodynamics directly to the movement of the shower particles.

The influence of several aspects of the calculation have already been studied [3] and are summerized in table 1 . Here, we continue the investigation of the influence of approximations in simulations by studying the impact of a finite step-length. We also perform a detailed comparison of the two independent simulation codes CoREAS and ZHAireS [4]. Both codes use different formalisms for the calculation of the radio emission. In CoREAS the "endpoint formalism" is implemented, in ZHAireS we use the time-domain implementation of the "ZHS algorithm". The numerical implementation of those algorithms might add additional uncertainties. A systematic study of the radio emission of both codes allows us to estimate the systematic uncertainty in the calculations of the radiation energy. This value is a universal quantity, valid for all air shower radio experiments. Measurement uncertainties recently decreased below the $10 \%$-level [5], consequently even minor uncertainties in the calculations are relevant.

^e-mail: gottowik@uni-wuppertal.de 


\begin{tabular}{lc}
\hline source of uncertainty & $\begin{array}{c}\text { uncertainty of } \\
\text { radiation energy }\end{array}$ \\
\hline hadronic interaction models & $0.3 \%$ \\
$\begin{array}{l}\text { approximations in the air-shower simulation } \\
\text { particle thinning }\end{array}$ & $<0.3 \%$ \\
energy thresholds of shower particles & $<1 \%$ \\
state of the atmosphere & $<3 \%$ \\
underlying first principle calculations & $<\mathbf{5 . 2 \%}$ \\
\hline
\end{tabular}

Table 1. Systematic uncertainties of the calculation of the radiation energy from the electromagnetic shower energy. Non-bold values taken from [3].

\section{Simulation Setup}

We simulate 1000 proton- and 1000 iron-induced air showers with each code. The primary energies follow a uniform distribution of the logarithmic energy between $10^{17} \mathrm{eV}$ and $10^{19} \mathrm{eV}$. The azimuth angle is distributed uniformly between $0^{\circ}$ and $360^{\circ}$ and the zenith angle uniformly between $0^{\circ}$ and $75^{\circ}$. We use the same description of the atmosphere, refractivity and hadronic interaction model in both codes. The geomagnetic field and the refractivity at sea level correspond to the environmental conditions of the Engineering Radio Array of the Pierre Auger Observatory [6]. The CoREAS traces are zero-padded to match the length of the ZHAireS traces. This ensures an identical bandwidth for the filtering to the $30-80 \mathrm{MHz}$ frequency band, the typical band used in cosmic-ray radio detectors today.

We only simulate antennas on the $\mathbf{v} \times(\mathbf{v} \times \mathbf{B})$ axis $(\mathbf{v}$ : shower axis, $\mathbf{B}$ : magnetic field) in the showerplane. Then, $E_{\text {rad }}$ can be computed as an integral of the energy fluence $f$ as

$$
E_{\mathrm{rad}}=2 \pi \int_{0}^{\infty} \mathrm{d} r r f(r)
$$

We correct $E_{\text {rad }}$ for effects due to geomagnetic angle $\alpha$ and atmospheric density at the shower maximum $\rho\left(X_{\max }\right)$ and obtain the corrected radiation energy (as described in [3]) $S_{\text {rad }}$ via

$$
\begin{aligned}
S_{\mathrm{rad}}= & \frac{E_{\mathrm{rad}}}{a\left(\rho\left(X_{\max }\right)\right)^{2}+\left(1-a\left(\rho\left(X_{\max }\right)\right)^{2}\right) \sin ^{2} \alpha} \\
& \cdot \frac{1}{\left(1-p_{0}+p_{0} \exp \left[p_{1}\left(\rho\left(X_{\max }\right)-\rho\left(\left\langle X_{\max }\right\rangle\right)\right)\right]\right)^{2}},
\end{aligned}
$$

with a parametrized charge-excess fraction $a\left(\rho\left(X_{\max }\right)\right)$.

\section{Results}

We find that $E_{\text {rad }}$ increased by $11 \%$ with a decrease in step size in CORSIKA, i.e. reducing the "STEPFC" parameters from default value 1 to 0.05 (cf. Fig 1). Previous comparisons between measured data and CoREAS simulations might improve by taking this modification into account. A 


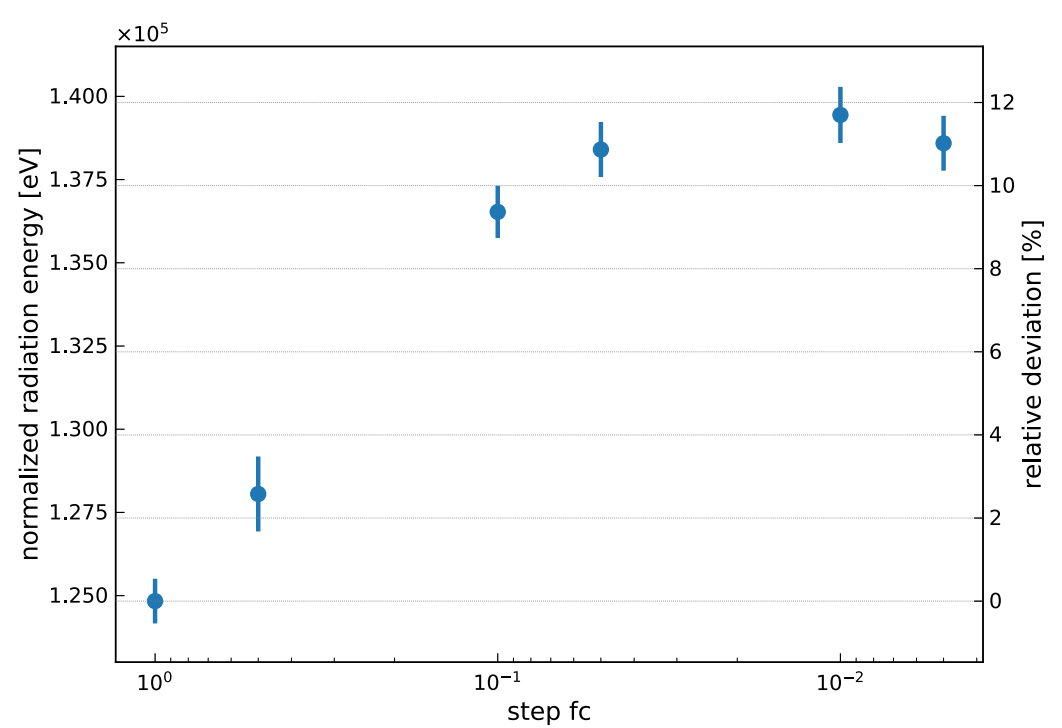

Figure 1. Influence of the STEPFC parameter of CORSIKA on the radiation energy for a $10^{17} \mathrm{eV}$ air shower simulated with CoREAS. Shown is the absolute value and the relative deviation from the default setting. The errorbar shows the uncertainty of the mean radiation energy of 40 air shower simulations.

comparison of the step sizes between both codes reveals slightly larger steps for ZHAireS at low altitudes. From the maximal observed differences we estimate a possible increase of $E_{\text {rad }}$ for ZHAireS by approximately $1 \%$ if smaller step sizes were used.

The simulation setup allows to disentangle the contribution of both radio emission mechanisms and to calculate the charge-excess fraction for both codes as a function of the density at the shower maximum. We find a good agreement between CoREAS and ZHAireS. With the data of both codes we obtain a combined parametrization for the charge excess fraction. We fit Eq. 2 and a power law

$$
S_{\mathrm{rad}}=A \cdot 10^{7} \mathrm{eV}\left(E_{\mathrm{em}} / 10^{18} \mathrm{eV}\right)^{B}
$$

to obtain the parameters $p_{0}$ and $p_{1}$ of Eq. 2 . With the combined parametrizations we correct $E_{\mathrm{rad}}$ and fit the power law individually for each code. We find $B$ equal to two as expected for coherent emission and a $5.2 \%$ larger value for $A$ with CoREAS in comparison to ZHAireS. If we interpret this deviation as a systematic uncertainty on the absolute prediction of the radiation energy we obtain an uncertainty in the determination of the absolute scale of the electromagnetic energy of $2.6 \%$. Thus, Monte Carlo predictions of the radiation energy of extensive air showers in the $30-80 \mathrm{MHz}$ band can be used to set an accurate absolute energy scale for cosmic ray detectors.

\section{References}

[1] A. Aab et al. (Pierre Auger Collaboration), Phys. Rev. Lett. 116, 241101 (2016)

[2] A. Aab et al. (Pierre Auger Collaboration), Phys. Rev. D93, 122005 (2016)

[3] C. Glaser, M. Erdmann, J.R. Hörandel, T. Huege, J. Schulz, JCAP 1609, 024 (2016), 1606. 01641

[4] M. Gottowik, C. Glaser, T. Huege, J. Rautenberg, Astroparticle Physics in press (2018), https://doi.org/10.1016/j . astropartphys.2018.07.004

[5] A. Aab et al. (Pierre Auger Collaboration), JINST 12, T10005 (2017)

[6] J. Rautenberg for the Pierre Auger Collaboration, Radio detection of ultra high energy cosmic rays with the Auger Engineering Radio Array, in this proceedings (2018) 